\title{
Estimación de estado de una unidad de craqueo catalítico fluido con filtro desaromatizado de Kalman
}

\section{State estimation of a FCCU with Unscented Kalman Filter}

\section{Cepero-Díaz Ariel Omar}

Universidad Tecnológica de La Habana José Antonio Echeverría, Cuba

Correo: arielc@automatica.cujae.edu.cu
González-Santos Ana Isabel

Universidad Tecnológica de La Habana José Antonio Echeverría, Cuba Correo: anita@automatica.cujae.edu.cu

\section{Resumen}

La medición directa de muchas variables en procesos industriales adiciona complejidad y costos a los sistemas y no garantiza buenos resultados en algunas ocasiones. Esto ha motivado el desarrollo de los estimadores de estado. En este trabajo se presenta un filtro de Kalman desaromatizado (UKF) para estimar algunas variables que no se miden en una unidad de craqueo catalítico fluido (FCCU). Este estimador se basa en un modelo de caja gris de la FCCU, presentado también en el artículo, que combina un modelo complejo de conocimiento con redes neuronales.

Descriptores: estimadores de estados, modelado, simulación, unidad de craqueo.

\begin{abstract}
The direct measurement of many variables in industrial processes adds complexity and cost to the system and it doesn't give good results sometimes. This has motivated the development of state estimators. In this paper is presented an Unscented Kalman Filter (UKF) for estimating different variables in a fluid catalytic cracking unit (FCCU). This estimator is based on a grey box model of the FCCU, also presented in this paper, which combine a complex knowledge model with neural networks.

Keywords: state estimators, modeling, simulation, FCCU.
\end{abstract}

\section{INTRODUCCIÓN}

La unidad de craqueo catalítico fluido (FCCU, del inglés Fluid Catalytic Cracking Unit) es una de las unidades tecnológicas más importantes que se utilizan en la industria de refinación de petróleo. Su función principal es la de transformar, por medio de reacciones de craqueo catalítico, un producto de masa molecular alta en otros de menor masa y puede decirse que es la base de la refinación moderna (Ramachandran et al., 2007). La reacción de craqueo de hidrocarburos consiste en la ruptura de los enlaces entre átomos de carbono en moléculas de hidrocarburos de masa molecular alta, cuando su temperatura se eleva a valores aproximados entre
925 K y 1035 K. Como resultado de la reacción se obtienen coque y una mezcla de hidrocarburos cuyas moléculas son de menor masa molecular y de mayor valor de uso. El término de craqueo catalítico se refiere al caso en que la reacción de craqueo ocurre en presencia de un catalizador que facilita o acelera la misma. En una FCCU esta reacción ocurre continuamente en un reactor en el que se ponen en contacto una corriente de hidrocarburos pesados y una corriente de catalizador que fluye mezclado con vapor (Sadeghbeigi, 2000).

La FCCU está compuesta por varios subsistemas: el horno de precalentamiento del producto a procesar, el reactor de craqueo, el regenerador de catalizador, los sopladores de aire, la torre fraccionadora de los pro- 
ductos resultantes, el compresor de gases húmedos y los conductos del catalizador (Sadeghbeigi 2000). En la Figura 1 se muestra un esquema general de una FCCU. También forman parte de la FCCU una serie de equipos auxiliares, instrumentos de medición y medios técnicos de automatización. El producto a procesar, proveniente de los tanques de almacenamiento y establecido en el esquema de la Figura 1 como flujo de alimentación, se precalienta en el horno hasta la temperatura adecuada para el proceso de craqueo. En el reactor se ponen en contacto el catalizador a temperaturas altas y el producto precalentado en el horno, produciéndose la reacción de craqueo catalítico. Los gases resultantes de la reacción salen por el tope del reactor hacia la torre fraccionadora, mientras que el catalizador gastado, con el coque y restos de hidrocarburos resultantes de la reacción adheridos a este, fluye por el conducto curvo inferior correspondiente hacia el regenerador, ayudado del aire suministrado por el soplador de aire de elevación. En el regenerador se restaura la actividad del catalizador gastado mediante la combustión del coque y de los restos de hidrocarburos depositados sobre el mismo en presencia del aire suministrado por el soplador de aire de combustión. El catalizador regenerado, y con una alta temperatura producto de la propia reacción de combustión, fluye de regreso hacia el reactor por el conducto curvo inferior correspondiente. En la torre fraccionadora se elimina el exceso de calor del producto resultante que proviene del tope del reactor mediante la recirculación de cuatro fracciones de los productos resultantes del craqueo. Finalmente los productos del craqueo pasan de la torre fraccionadora hacia el compresor de gas húmedo, que los comprime e impulsa hacia la unidad recolectora, donde se separarán en productos finales ligeros (Sadeghbeigi, 2000).

La FCCU se caracteriza por ser un sistema multivariable con una dinámica acentuadamente no lineal, con gran interacción entre sus variables y una compleja cinética en las reacciones de craqueo y combustión de coque, por ello su modelado y control resultan muy complejos (McFarlane et al., 1993). Numerosos trabajos

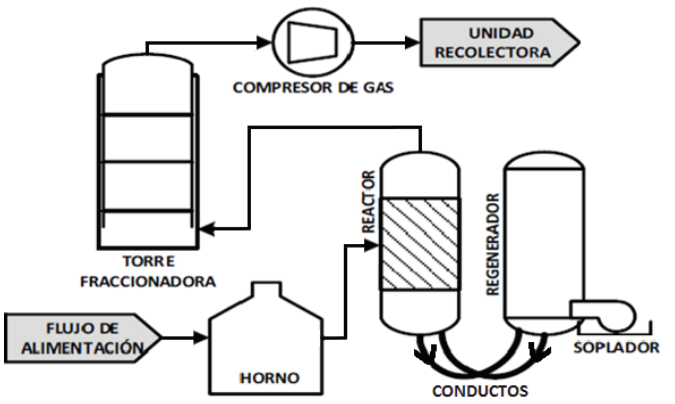

Figura 1. Esquema general de una FCCU se han publicado sobre el modelado de las FCCU, con una amplia variación de alcance y de rigor en el nivel de modelado (Ramachandran et al., 2007). Algunos trabajos se enfocan en partes específicas de las unidades (Souza et al., 2011; Zhang et al., 2012; Garcia-Dopico and Garcia, 2017), mientras que otros se ocupan solo de la cinética de las reacciones o el comportamiento en estado estacionario (Heydari et al., 2010; Dasila et al., 2012). Se han desarrollado modelos empíricos de las FCCU (Michalopoulos et al., 2001; Tootoonchy y Hashemi, 2013; Long et al., 2015), que responden adecuadamente a unidades reales en un estrecho rango de operación, y modelos de primeros principios (McFarlane et al., 1993; Han y Chung 2001), más generales, pero con un elevado número de ecuaciones diferenciales y algebraicas, algunas de las cuales contienen simplificaciones importantes de los fenómenos descritos.

Otra característica de las FCCU es que no todas las variables que conforman el vector de estado en los modelos de caja blanca reportados son medibles, por lo que se hace necesario el uso de un estimador de estados para la implementación de las técnicas avanzadas de control que se basan en el conocimiento del estado del sistema. En Boum et al. (2015) se presenta un estimador de tipo filtro extendido de Kalman para una FCCU, cuyas estimaciones del estado y parámetros del modelo de la FCCU se emplean como parte de una estrategia de control multivariable, mientras que en Roman et al. (2009) se presenta un estimador de tipo estimador de horizonte deslizante de una FCCU, que luego se emplea dentro de un esquema de control predictivo no lineal.

\section{Modelo GRIS DE UNA FCCU DE TIPO IV}

Ante la dificultad práctica para obtener el valor de algunos de los parámetros implicados en los modelos de caja blanca de la FCCU de tipo IV reportados, se propone desarrollar un modelo gris de la unidad. Para ello se tomó como referencia el modelo de una FCCU de tipo IV que presentó el trabajo de McFarlane y colaboradores (1993) y que ha servido también como modelo de prueba para otros trabajos reportados en la literatura (Ellis et al., 1998; Huang et al. 2000; Garcia-Dopico and Garcia, 2017). Este modelo de referencia agrupa 117 ecuaciones entre algebraicas y diferenciales donde se relacionan un total de 17 variables de entrada, 20 variables de salida y 15 variables de estado. El modelo gris propuesto conserva estas mismas variables, las cuales se listan en la Tabla 1, Tabla 2 y Tabla 3, respectivamente. Además, existe un conjunto de 13 variables auxiliares algebraicas explícitas en la conexión de los diferentes 
bloques que componen el modelo gris propuesto, las cuales se listan en la Tabla 4.

El modelo gris que se propone conserva el modelo de referencia, como parte de caja blanca, las ecuaciones algebraicas y diferenciales que modelan los aspectos del funcionamiento de la FCCU que obedecen a principios claramente determinados, las cuales se corresponden con las ecuaciones (1) a la (34) que se agrupan por subsistema.

Tabla 1. Variables de entrada del modelo gris de la FCCU

\begin{tabular}{lc}
\hline \multicolumn{1}{c}{ Variable } & Símbolo \\
\hline Posición de la válvula de succión del compresor de gas húmedo (0-1) & $V 11$ \\
Posición de la válvula de escape de gas húmedo (0-1) & $V 12$ \\
Posición de la válvula de bypass de gas húmedo (0-1) & $V 13$ \\
Posición de la válvula de los gases de combustión (0-1) & $V 14$ \\
Posición de la válvula de succión del soplador de aire de combustión (0-1) & $V 6$ \\
Posición de la válvula de escape de aire de combustión (0-1) & $V 7$ \\
Posición de la válvula de escape de aire de elevación (0-1) & $V 8$ \\
Posición de la válvula de reboso de aire de elevación (0-1) & $V 10$ \\
Posición de la válvula de vapor del soplador de aire de elevación (0-1) & $V l$ \\
Temperatura de la alimentación fresca que entra al horno (K) & $T 1$ \\
Flujo de combustible para el horno (m³/s) & $F 5$ \\
Flujo de aceite de lavado $(\mathrm{kg} / \mathrm{s})$ & $F 1$ \\
Flujo de diésel (kg/s) & $F 2$ \\
Flujo de gasóleo $(\mathrm{kg} / \mathrm{s})$ & $F 3$ \\
Flujo de lodo líquido reciclado $(\mathrm{kg} / \mathrm{s})$ & $F 4$ \\
Factor efectivo de formación de coque del alimentado & $\mathrm{Ff}$ \\
Temperatura atmosférica $(\mathrm{K})$ & $\mathrm{Ta}$ \\
\hline
\end{tabular}

Tabla 2. Variables de salida del modelo gris de la FCCU

\begin{tabular}{|c|c|}
\hline Variable & Símbolo \\
\hline Flujo de succión del soplador de aire de combustión $(\mathrm{kg} / \mathrm{s})$ & F6 \\
\hline Flujo de aire para combustión hacia el regenerador $(\mathrm{kg} / \mathrm{s})$ & F7 \\
\hline Flujo de succión del soplador de aire de elevación $(\mathrm{kg} / \mathrm{s})$ & F8 \\
\hline Flujo de aire de elevación hacia el regenerador $(\mathrm{kg} / \mathrm{s})$ & F9 \\
\hline Flujo de reboso de aire de elevación hacia el regenerador $(\mathrm{kg} / \mathrm{s})$ & $F 10$ \\
\hline Presión de succión del soplador de aire de combustión ( $\mathrm{kPa})$ & $P 1$ \\
\hline Velocidad del soplador de aire de elevación ( $\mathrm{rad} / \mathrm{s})$ & sa \\
\hline Concentración de CO en los gases de la chimenea (ppm) & $c \mathrm{CO}$ \\
\hline Concentración de $\mathrm{CO}_{2}$ en los gases de la chimenea (ppm) & $c \mathrm{CO}_{2}$ \\
\hline Concentración de $\mathrm{O}_{2}$ en los gases de la chimenea (\%) & $\mathrm{cO}_{2}$ \\
\hline Temperatura de los gases de la combustión a la salida de los ciclones $(\mathrm{K})$ & Tc \\
\hline Temperatura en el regenerador $(\mathrm{K})$ * & $\operatorname{Trg}$ \\
\hline Presión en el regenerador $(\mathrm{kPa})$ * & P6 \\
\hline Nivel de catalizador en el pozo de reboso del regenerador (m) & Lsp \\
\hline Presión en el reactor $(\mathrm{kPa})$ & P4 \\
\hline Temperatura en el elevador del reactor $(\mathrm{K})$ * & $\operatorname{Tr}$ \\
\hline Presión en el tope de la torre fraccionadora $(\mathrm{kPa}) *$ & P5 \\
\hline Flujo de succión de gas húmedo en el compresor $(\mathrm{kmol} / \mathrm{s})$ & $F 11$ \\
\hline Temperatura de la alimentación fresca que entra al elevador del reactor $(\mathrm{K})$ * & $T 2$ \\
\hline Temperatura en la caja de fuego del horno $(\mathrm{K})$ * & T3 \\
\hline${ }^{*}$ son variables de estado también & \\
\hline
\end{tabular}


DOI: http://dx.doi.org/10.22201/fi.25940732e.2018.19n1.002

EstimaCión DE ESTADO dE UNA UNIDAD DE CRAQUEO CATALÍTICO FLUIDO CON FILTRO DESAROMATIZADO DE KALMAN

Tabla 3. Variables de estado del modelo gris de la FCCU

\begin{tabular}{|c|c|}
\hline Variable & Símbolo \\
\hline Fracción másica de carbono en el catalizador regenerado (kg/kg) & $\mathrm{Crgc}$ \\
\hline Fracción másica de coque en el catalizador usado (kg/kg) & $\operatorname{Csc}$ \\
\hline Presión de descarga del soplador de aire de combustión $(\mathrm{kPa})$ & $P 2$ \\
\hline Presión de descarga del soplador de aire de elevación $(\mathrm{kPa})$ & P3 \\
\hline Cantidad de gas en el regenerador (mol) & $n$ \\
\hline Masa del catalizador en el regenerador $(\mathrm{kg})$ & Wrg \\
\hline Masa del catalizador en el pozo de reboso del regenerador $(\mathrm{kg})$ & Wsp \\
\hline Temperatura en el regenerador $(\mathrm{K})$ * & $\operatorname{Trg}$ \\
\hline Presión en el regenerador $(\mathrm{kPa}) *$ & P6 \\
\hline Masa del catalizador en el reactor $(\mathrm{kg})$ & $W r$ \\
\hline Temperatura en el elevador del reactor $(\mathrm{K})$ * & $\operatorname{Tr}$ \\
\hline Presión en el tope de la torre fraccionadora $(\mathrm{kPa})^{*}$ & $P 5$ \\
\hline Presión de succión en el compresor de gas húmedo (kPa) & $P 7$ \\
\hline Temperatura de la alimentación fresca que entra al elevador del reactor $(\mathrm{K})$ * & $T 2$ \\
\hline Temperatura en la caja de fuego del horno $(\mathrm{K}){ }^{*}$ & T3 \\
\hline * son variables de salida también & \\
\hline
\end{tabular}

Tabla 4. Variables auxiliares algebraicas del modelo gris de la FCCU

\begin{tabular}{lc}
\hline \multicolumn{1}{c}{ Variable } & Símbolo \\
\hline Flujo de catalizador regenerado en el conducto curvo $(\mathrm{kg} / \mathrm{s})$ & Frgc \\
Flujo de catalizador usado en el conducto curvo $(\mathrm{kg} / \mathrm{s})$ & $F s c$ \\
Flujo de catalizador hacia el pozo de reboso del regenerador $(\mathrm{kg} / \mathrm{s})$ & $F s p$ \\
Razón de combustión de hidrógeno $(\mathrm{kg} / \mathrm{s})$ & $F h$ \\
Flujo de gas húmedo producido en el reactor $(\mathrm{kmol} / \mathrm{s})$ & Fwg \\
Flujo de gases de escape a la chimenea $(\mathrm{kmol} / \mathrm{s})$ & $F s g$ \\
Flujo total de aire suministrado al regenerador $(\mathrm{kmol} / \mathrm{s})$ & Fair \\
Relación molar de $\mathrm{CO}$ en los gases de la chimenea $(\mathrm{mol} / \mathrm{mol})$ & $x C O$ \\
Relación molar de $\mathrm{CO}_{2}$ en los gases de la chimenea $(\mathrm{mol} / \mathrm{mol})$ & $x C \mathrm{O}_{2}$ \\
Relación molar de $\mathrm{O}_{2}$ en los gases de la chimenea $(\mathrm{mol} / \mathrm{mol})$ & $x \mathrm{O}_{2}$ \\
Presión en el fondo del elevador del reactor $(\mathrm{kPa})$ & $\mathrm{Prb}$ \\
Presión en el fondo del regenerador $(\mathrm{kPa})$ & $\mathrm{Prgb}$ \\
Presión en el fondo del elevador del regenerador $(\mathrm{kPa})$ & $\mathrm{Pblp}$ \\
\hline
\end{tabular}

\section{REACTOR, PARTE DE CAJA BLANCA}

Wris $=\frac{F r g c \cdot \text { Aris } \cdot \text { hris } \cdot \rho v \cdot \rho p}{(F 3+F 4) \cdot \rho p+F r g c \cdot \rho v}$

$\operatorname{tr}=\frac{\text { Wris }}{60 \cdot F r g c}$

$W H S V=3600 \frac{F 3+F 4}{\text { Wris }}$

$$
F c k=0.5852 F 1 \frac{(f f \cdot F 3+5 \cdot F 4+3 \cdot F 1-0.8 \cdot F 2) \cdot t r^{-0.69}}{W H S V}
$$

$$
P r b=P 4+\frac{(F 3+F 4+F r g c) \cdot h r i s \cdot \rho v \cdot \rho p \cdot g}{1000 \cdot((F 3+F 4) \cdot \rho p+F r g c \cdot \rho v)}
$$

$$
\frac{d C s c}{d t}=\frac{F r g c \cdot(C r g c-C s c)+F c k}{W r}
$$




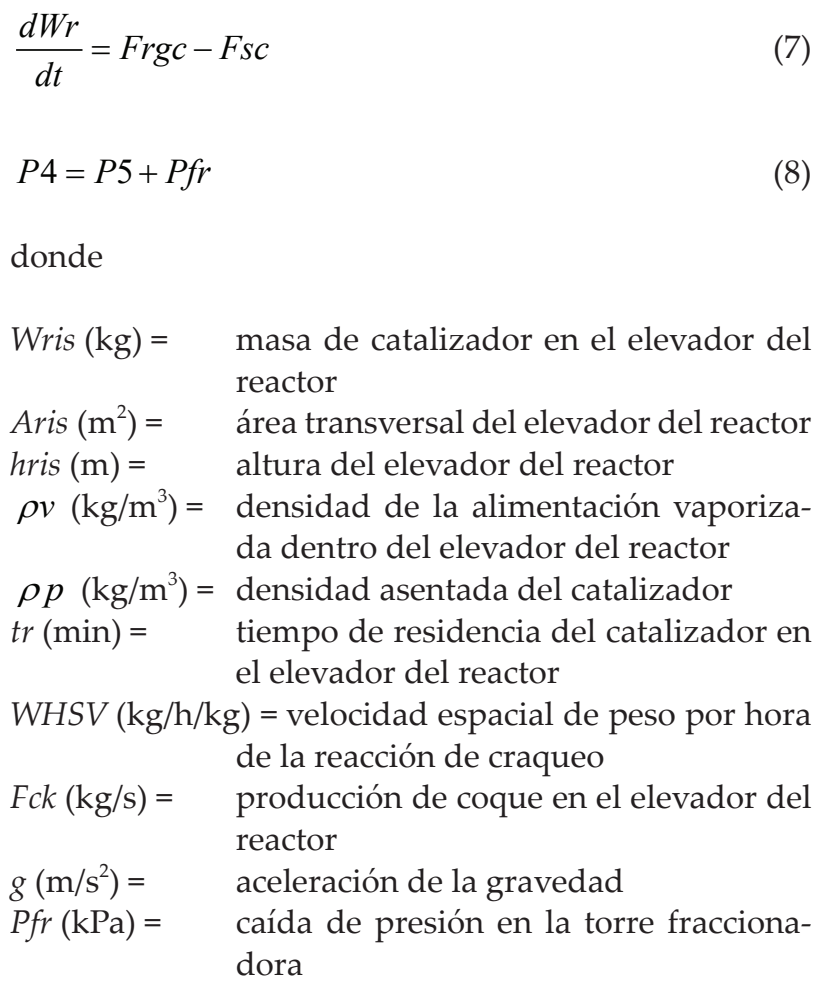

\section{Regenerador, PARTE de Caja blanCa}

$L s p=\frac{W s p}{\rho c \cdot A s p}$

$F h=F s c \cdot(C s c-C r g c) \cdot C h$

$\frac{d W s p}{d t}=F s p-F r g c$

$\frac{d W r g}{d t}=F s c-F s p$

$\frac{d C r g c}{d t}=\frac{F s c \cdot(C s c-C r g c)-F h-12 \cdot F a i r\left(x C O+x C_{2}\right)}{W r g}$

$\operatorname{Prgb}=P 6+\frac{\mathrm{Wrg} \cdot \mathrm{g}}{1000 \cdot \mathrm{Arg}}$

$c \mathrm{CO}=\frac{28 \cdot 10^{6} \cdot x \mathrm{CO}}{28 \cdot x \mathrm{CO}+44 x \mathrm{CO}_{2}+32 \cdot x \mathrm{O}_{2}+22.12}$

$$
\begin{aligned}
& { }_{c \mathrm{CO}_{2}}=\frac{\mathrm{Fair} \cdot x \mathrm{CO}_{2} \cdot 100}{F_{s g}} \\
& c \mathrm{O}_{2}=\frac{\text { Fair } \cdot x \mathrm{O}_{2} \cdot 100}{F_{s g}}
\end{aligned}
$$

donde

$\rho c\left(\mathrm{~kg} / \mathrm{m}^{3}\right)=$ densidad del catalizador en el pozo de reboso del regenerador

Asp $\left(\mathrm{m}^{2}\right)=\quad$ área transversal del pozo de reboso del regenerador

$C h=\quad$ fracción másica de hidrógeno en el co$\operatorname{Arg}\left(\mathrm{m}^{2}\right)=\quad$ área transversal del regenerador

\section{Conductos Curvos, PARTE DE CAJA BLANCA}

$\Delta \operatorname{Prg} c=1000 \cdot(P 6-P r b)+\frac{W s p \cdot g}{A s p}+(E t-E o) \cdot \rho c \cdot g$

$$
F r g c=\frac{\Delta \operatorname{Prgc} \cdot \rho c \cdot A r g c^{2}}{\operatorname{Lrgc} \cdot \operatorname{frgc}}
$$

$\Delta P s c=1000 \cdot(P 4-P b l p)+\frac{W r \cdot g}{A s}+(E s-E l) \cdot \rho c \cdot g$

$F s c=\frac{\Delta P s c \cdot \rho c \cdot A s c^{2}}{L s c \cdot f s c}$

donde

$\Delta \operatorname{Prgc}(\mathrm{Pa})=$ diferencia de presión en el conducto de catalizador regenerado

$\Delta P S c(\mathrm{~Pa})=\quad$ diferencia de presión en el conducto de catalizador usado

$E t(\mathrm{~m})=\quad$ altura de la toma del conducto de catalizador regenerado en el pozo de reboso del regenerador

Eo $(\mathrm{m})=\quad$ altura de la toma del conducto de catalizador regenerado en el elevador del reactor

$E s(\mathrm{~m})=\quad$ altura de la toma del conducto de catalizador usado en el separador del reactor 


\begin{tabular}{|c|c|}
\hline$E l(\mathrm{~m})$ & $\begin{aligned}= & \text { altura de la toma del conducto de } \\
& \text { catalizador usado en el elevador } \\
& \text { del regenerador }\end{aligned}$ \\
\hline $\operatorname{Argc}\left(\mathrm{m}^{2}\right)$ & $\begin{aligned}= & \text { área transversal del conducto } \\
& \text { de catalizador regenerado }\end{aligned}$ \\
\hline$A s c\left(\mathrm{~m}^{2}\right)$ & $\begin{aligned}= & \text { área transversal del conducto de } \\
& \text { catalizador usado }\end{aligned}$ \\
\hline As $\left(\mathrm{m}^{2}\right)$ & $\begin{aligned}= & \text { área transversal del separador del } \\
& \text { reactor }\end{aligned}$ \\
\hline $\operatorname{Lrgc}(\mathrm{m})$ & $\begin{aligned}= & \text { longitud del conducto de cataliza- } \\
& \text { dor regenerado }\end{aligned}$ \\
\hline $\operatorname{Lsc}(\mathrm{m})$ & $\begin{aligned}= & \text { longitud del conducto de cataliza- } \\
& \text { dor usado }\end{aligned}$ \\
\hline $\operatorname{frgc}(\mathrm{Pa} \cdot \mathrm{s})$ & $\begin{aligned}= & \text { factor de fricción en el conducto de } \\
& \text { catalizador regenerado }\end{aligned}$ \\
\hline$f_{S C}(\mathrm{~Pa} \cdot \mathrm{s})$ & $\begin{aligned}= & \text { factor de fricción en el conducto de } \\
& \text { catalizador usado }\end{aligned}$ \\
\hline
\end{tabular}

TORRE FRACCIONADORA Y COMPRESOR DE GASES HÚMEDOS, PARTE DE CAJA BLANCA

$$
\begin{aligned}
& F V 11=\left\{\begin{array}{r}
k 11 \cdot 0.0225^{(1-V 11)} \cdot \sqrt{P 5-P 7}, V 11>0.5 \\
k 11 \cdot 0.3 \cdot V 11 \cdot \sqrt{P 5-P 7}, V 11 \leq 0.5
\end{array}\right\} \\
& F V 12=k 12 \cdot V 12 \cdot \sqrt{P 5-P a} \\
& F V 13=k 13 \cdot V 13 \cdot P v r u \\
& \frac{d P 5}{d t}=12.64 \cdot(F w g-F V 11-F V 12+F V 13) \\
& \frac{d P 7}{d t}=75.84 \cdot(F V 11-F 11)
\end{aligned}
$$

donde

$$
\begin{aligned}
k 11\left(\mathrm{kmol} / \mathrm{s} /(\mathrm{kPa})^{1 / 2}\right)= & \begin{array}{l}
\text { ganancia de la válvula de suc } \\
\text { ción del compresor de gases hú } \\
\text { medos }
\end{array} \\
k 12\left(\mathrm{kmol} / \mathrm{s} /(\mathrm{kPa})^{1 / 2}\right)= & \begin{array}{l}
\text { gananciade la válvula de escape } \\
\text { de gas húmedo }
\end{array} \\
k 13\left(\mathrm{kmol} / \mathrm{s} /(\mathrm{kPa})^{1 / 2}\right)= & \begin{array}{l}
\text { ganancia de la válvula de bypass } \\
\text { de gas húmedo }
\end{array} \\
= & \text { flujo a través de la válvula de } \\
& \text { succión del compresor de gases } \\
& \text { húmedos } \\
= & \text { flujo a través de la válvula de es } \\
& \text { cape de gas húmedo }
\end{aligned}
$$

FV13 $(\mathrm{kmol} / \mathrm{s})=$ flujo a través de la válvula de bypass de gas húmedo
Pvru $(\mathrm{kPa}) \quad=$ presión en la unidad recuperadora de vapor
$\mathrm{Pa}(\mathrm{kPa}) \quad=$ presión atmosférica

\section{Sopladores de AIRE, parte de CAJA blanCA}

$F V 7=\left\{\begin{array}{cc}k 7 \cdot 0,0225^{(1-V 7)} \cdot \sqrt{P 2-P a} & , V 7>0,5 \\ k 7 \cdot 0,3 \cdot V 7 \cdot \sqrt{P 2-P a} & , V 7 \leq 0,5\end{array}\right\}$

$F 7=k c \cdot \sqrt{P 2-P r g b}$

$\frac{d P 2}{d t}=\frac{R \cdot T c d}{29 \cdot V c d} \cdot(F 6-F V 7-F 7)$

$F 10=k 10 \cdot V 10 \cdot \sqrt{P 3-P r g b}$

$F V 8=k 8 \cdot V 8 \cdot \sqrt{P 3-P a}$

$$
F 9=k l \cdot \sqrt{P 3-P b l p}
$$

$\frac{d P 3}{d t}=\frac{R \cdot T l d}{29 \cdot V l d} \cdot(F 8-F V 8-F 9-F 10)$

Fair $=\frac{F 7+F 9+F 10}{29}$

donde

$k 7\left(\mathrm{~kg} / \mathrm{s} /(\mathrm{kPa})^{1 / 2}\right)$ = ganancia de la válvula de escape de aire de combustión

$k 8\left(\mathrm{~kg} / \mathrm{s} /(\mathrm{kPa})^{1 / 2}\right)=$ ganancia de la válvula de escape de aire de elevación

$k 10\left(\mathrm{~kg} / \mathrm{s} /(\mathrm{kPa})^{1 / 2}\right)=$ ganancia de la válvula de reboso de aire de elevación

$k c\left(\mathrm{~kg} / \mathrm{s} /(\mathrm{kPa})^{1 / 2}\right)=$ resistencia al flujo en la tubería de aire de combustión

$k l\left(\mathrm{~kg} / \mathrm{s} /(\mathrm{kPa})^{1 / 2}\right)=$ resistencia al flujo en la tubería de aire de elevación

$F V 7(\mathrm{~kg} / \mathrm{s}) \quad=$ flujo a través de la válvula de escape de aire de combustión

$F V 8(\mathrm{~kg} / \mathrm{s}) \quad=$ flujo a través de la válvula de escape de aire de elevación 

$\operatorname{Tcd}(\mathrm{K})$
$\operatorname{Vcd}\left(\mathrm{m}^{3}\right)$
Tld $(\mathrm{K})$
$\operatorname{Vld}\left(\mathrm{m}^{3}\right)$
$R(\mathrm{~J} / \mathrm{K} / \mathrm{mol})$
= temperatura de descarga del sopla- dor de aire de combustión
= volumen de descarga del soplador de aire de combustión
= temperatura de descarga del sopla- dor de aire de elevación

En la parte de caja negra del modelo gris propuesto se modela, a través de seis redes neuronales dinámicas de tipo no lineal autorregresiva con variable externa (NARX) y 13 redes neuronales estáticas de tipo Perceptrón multicapa (MLP), los aspectos del funcionamiento de la FCCU que no obedecen a principios claramente determinados de acuerdo con el modelo de referencia. En la Tabla 5 se detallan el tipo, las entradas y la salida de cada una de las redes neuronales. En el diagrama en bloques de la Figura 2 se muestra la arquitectura del modelo gris propuesto, el cual se conforma por cinco bloques de caja negra y cinco de caja blanca y cuyos vectores de entrada, de estado y de salida se dan por (35), (36) y (37), respectivamente.

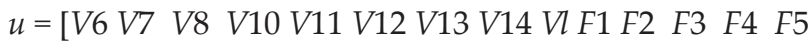

$$
\begin{aligned}
& \text { Ta T1 ff }]^{\mathrm{T}}
\end{aligned}
$$

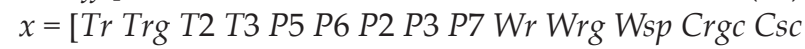

$$
\begin{aligned}
& n]^{\mathrm{T}}
\end{aligned}
$$

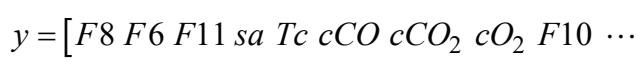$$
\text { ․ F7 F9 Lsp P4 P1 Tr Trg T2 T3 P5 P6 }]^{\mathrm{T}}
$$

\section{Filtro de Kalman desaromatizado (UKF)}

El UKF es un estimador probabilístico que utiliza la transformación desaromatizada para propagar la media y la covarianza del estado del sistema a través de las funciones no lineales del sistema (Julier y Uhlmann, 2004). En el UKF la distribución de probabilidad del estado se aproxima utilizando un conjunto mínimo de puntos de muestreo elegidos cuidadosamente, llamados puntos sigma, cuya media $\bar{x}$ y covarianza $P$ son los del estado. Las perturbaciones del estado $\varepsilon_{k}$ y los ruidos de la salida $v_{k}$ se supone que deben ser ruidos blancos no correlacionados con media cero y covarianza $Q$ y $R$, respectivamente.

Tabla 5. Redes neuronales que constituyen la parte de caja negra del modelo gris

\begin{tabular}{|c|c|c|c|}
\hline Subsistema & Tipo & Entradas & Salida \\
\hline \multirow{2}{*}{ Horno } & NARX & $F 5, F 3, T 1$ & $T 2$ \\
\hline & NARX & $F 5, F 3, T 1$ & T3 \\
\hline \multirow{2}{*}{ Reactor } & NARX & $\operatorname{Trg}, T 2, F 3, F 4, F r g C$ & $\operatorname{Tr}$ \\
\hline & MLP & $\operatorname{Tr}, F 3, F 4$ & Fwg \\
\hline \multirow{10}{*}{ Regenerador } & NARX & Tr, Fsc, Fair, Fh, Crgc, Frgc, V14, Wrg, Wsp & $\operatorname{Trg}$ \\
\hline & NARX & Tr, Fsc, Fair, Fh, Crgc, Frgc, V14, Wrg, Wsp & P6 \\
\hline & NARX & Tr, Fsc, Fair, Fh, Crgc, Frgc, V14, Wrg, Wsp & $n$ \\
\hline & MLP & Fair, Fh, Trg, Crgc, V14, Wrg, P6 & Tc \\
\hline & MLP & Fair, Fh, Trg, Crgc, V14, Wrg, P6 & $x \mathrm{O}_{2}$ \\
\hline & MLP & Fair, Fh, Trg, Crgc, V14, Wrg, P6 & $x C O$ \\
\hline & MLP & Fair, Fh, Trg, Crgc, V14, Wrg, P6 & $x \mathrm{CO}_{2}$ \\
\hline & MLP & Tr, Trg, Fsc, Fair, P3, P6, V14, Wrg & Pblp \\
\hline & MLP & Lsp, Trg, P6, Fair, V14, Wrg & Fsp \\
\hline & MLP & $P 6, V 14$ & Fsg \\
\hline $\begin{array}{l}\text { Torre y } \\
\text { Compresor }\end{array}$ & MLP & $P 7$ & $F 11$ \\
\hline \multirow{4}{*}{ Sopladores de aire } & MLP & $P 2, V 6, T a$ & $P 1$ \\
\hline & MLP & $P 2, V 6, T a$ & F6 \\
\hline & MLP & $V l$ & $s a$ \\
\hline & MLP & $V l, T a, P 3$ & $F 8$ \\
\hline
\end{tabular}


Un conjunto mínimo apropiado de $p=2 n+1$ puntos sigma y sus pesos asociados $S=\left\{x^{i}, s^{i}: i=0,1, \ldots, p\right\}$ se determina por (38), donde $n$ es la dimensión del vector de estado, $A^{j}$ es la j-ésima columna de la matriz raíz cuadrada de $P$, tal que $P=A A^{\mathrm{T}}$, y $\alpha$ es un factor de escala, tal que $n+\alpha \neq 0$, lo cual determina la dispersión de los puntos sigma alrededor de la media $\bar{x}$.

$$
\begin{array}{ll}
x^{0}=\bar{x} & s^{0}=\frac{\alpha}{n+\alpha} \\
x^{j}=\bar{x}+\sqrt{n+\alpha}(A)^{j} & s^{j}=\frac{1}{2(n+\alpha)} \\
x^{j+n}=\bar{x}-\sqrt{n+\alpha}(A)^{j} & s^{j+n}=\frac{1}{2(n+\alpha)} \\
j=1, . ., n &
\end{array}
$$

El algoritmo del estimador UKF, considerando perturbaciones $\varepsilon_{k}$ y ruidos $v_{k}$ aditivos, es el siguiente:

1. Generar el conjunto de puntos sigma y sus pesos asociados, usando la distribución dada por (38), a partir del estado estimado en la iteración anterior:

$$
\hat{\bar{x}}_{k-1}, \hat{P}_{k-1} \text {. }
$$

2. Propagar cada punto sigma al correspondiente nuevo punto sigma transformado a través de la ecuación de estado del sistema y teniendo en cuenta la entrada anterior del sistema $u_{k-1}$.

$$
\hat{x}_{k}^{j}=f\left(\hat{x}_{k-1}^{j}, u_{k-1}\right)
$$

3. Calcular la salida estimada del sistema correspondiente a cada nuevo punto sigma transformado a través de la ecuación de salida del sistema.

$$
\hat{y}_{k}^{j}=h\left(\hat{x}_{k}^{j}\right)
$$

4. Estimar la media a priori y la covarianza del estado actual.

$\hat{\bar{x}}_{k}^{-}=\sum_{j=0}^{2 n} s^{j} \hat{x}_{k}^{j}, \hat{P}_{k}^{-}=\sum_{j=0}^{2 n} s^{j}\left[\hat{x}_{k}^{j}-\hat{\bar{x}}_{k}^{-}\right]\left[\hat{x}_{k}^{j}-\hat{\bar{x}}_{k}^{-}\right]^{T}+Q$

5. Estimar la media a priori y la covarianza de la salida del sistema.

$\hat{\bar{y}}_{k}^{-}=\sum_{j=0}^{2 n} s^{j} \hat{y}_{k}^{j}, \hat{P}_{y, k}^{-}=\sum_{j=0}^{2 n} s^{j}\left[\hat{y}_{k}^{j}-\hat{\bar{y}}_{k}^{-}\right]\left[\hat{y}_{k}^{j}-\hat{\bar{y}}_{k}^{-}\right]^{T}+R$

6. Estimar la covarianza cruzada del estado actual y la salida del Sistema a priori.

$\hat{P}_{x y, k}^{-}=\sum_{j=0}^{2 n} s^{j}\left[\hat{x}_{\mathrm{k}}^{j}-\hat{\bar{x}}_{k}^{-}\right]\left[\hat{y}_{k}^{j}-\hat{\bar{y}}_{k}^{-}\right]^{T}$

7. Finalmente, estimar la media a posteriori y la covarianza del estado actual teniendo en cuenta la salida actual del sistema $y_{k}$.

$$
K_{k}=\hat{P}_{x y, k}^{-}\left[\hat{P}_{y, k}^{-}\right]^{-1}
$$

$\hat{\bar{x}}_{k}=\hat{\bar{x}}_{k}^{-}+K_{k}\left[y_{k}-\hat{\bar{y}}_{k}^{-}\right], \hat{P}_{k}=\hat{P}_{k}^{-}-K_{k} \hat{P}_{y, k}^{-} K_{k}{ }^{T}$

Con el objetivo de usar un modelo de caja gris del sistema a observar, los pasos 2 y 3 del algoritmo general se resuelven tomando en cuenta la estructura del modelo gris como se representa en la Figura 3, resultando en un filtro de Kalman desaromatizado basado en modelo gris (GMUKF). 


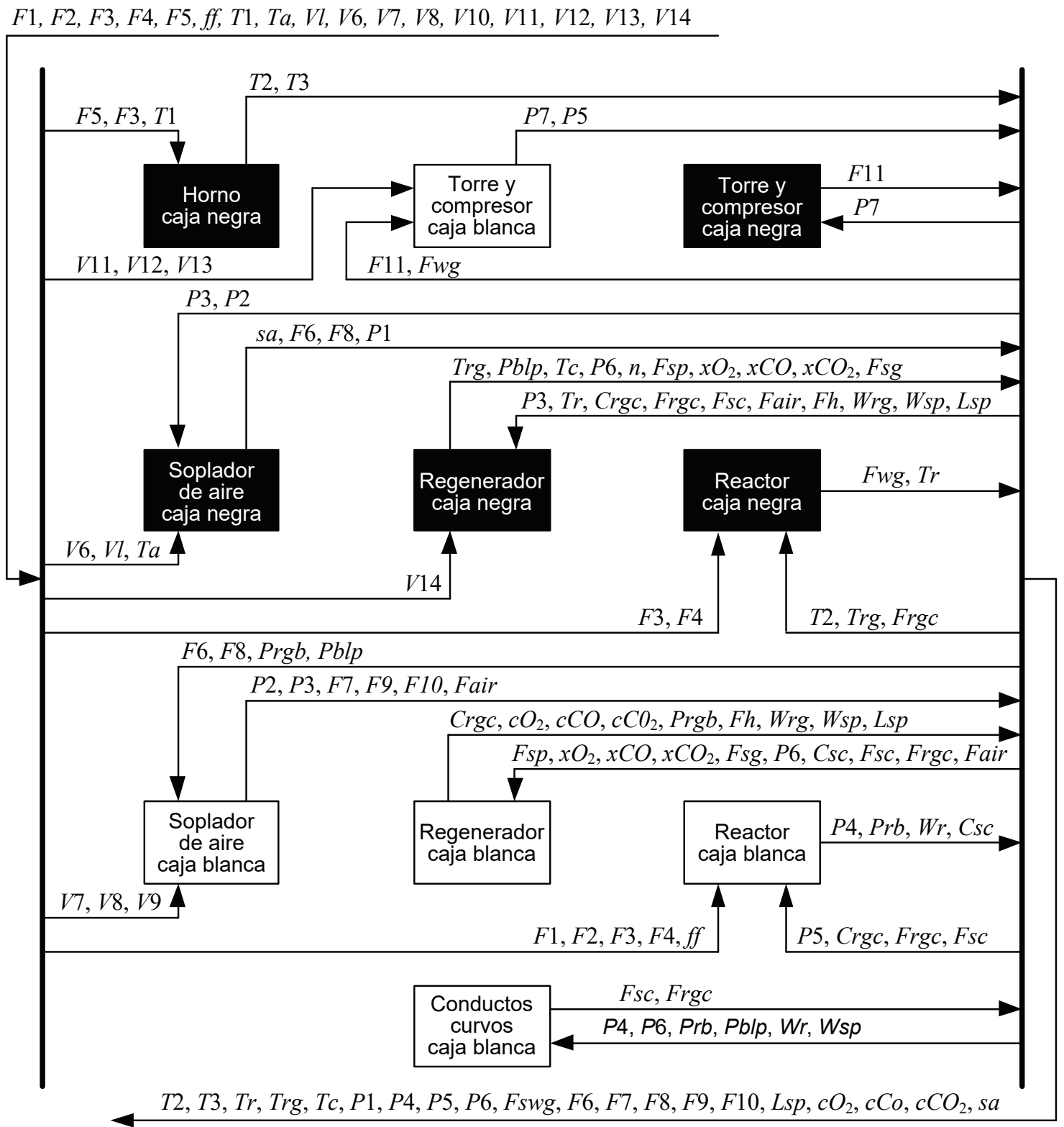

Figura 2. Modelo gris de la FCCU

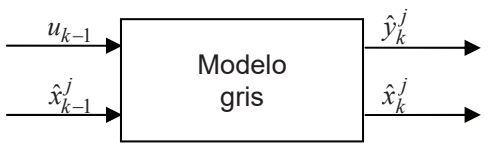

Figura 3. Uso del modelo gris en la formulación del UKF 


\section{Resultados del estimador GMUKF para UNA FCCU DE TIPO IV}

De las 15 variables de estado del sistema, seis se consideran a su vez como salidas del sistema, como se refleja en la Tabla 2 y Tabla 3, por tanto son medidas. Otras tres variables, que son $P 2, P 3$ y $P 7$, pueden medirse también sin dificultad $y$, aunque en el modelo no se declaran como salidas, puesto que existen otras variables de salidas directamente relacionadas con ellas, en las instalaciones industriales de las FCCU suelen colocarse instrumentos de medición e indicación local de las mismas. Las restantes seis variables de estado, que son $W r$, Wrg, Wsp, Crgc, Csc y n, no pueden medirse en la práctica de forma directa y continua, lo cual justifica el uso de un estimador de estados para conocer su valor. La disponibilidad del valor de estas variables de estado no medibles es importante para establecer técnicas de control avanzado en la FCCU, o para realizar la detección y diagnóstico de fallas en la unidad. También puede ser útil para determinar la calidad de las reacciones que ocurren en la unidad. Por ejemplo, el valor de la fracción másica de carbono en el catalizador regenerado, $\mathrm{Crgc}$, indica si la reacción de combustión en el regenerador está logrando regenerar suficientemente el catalizador o no. Comúnmente el valor de esta variable, Crgc, se obtiene por pruebas químicas de laboratorio a partir de una muestra de catalizador regenerado extraída del proceso, lo que conlleva un gasto de reactivos caros y la imposibilidad de conocer el valor de dicha variable de forma continua.

Se diseñó un estimador de tipo GMUKF para la FCCU, empleando el modelo gris descrito en la sección anterior, con las siguientes características: los puntos sigma se generan de acuerdo con la distribución dada por (37), el peso asociado al punto sigma central se ajustó como $s^{(0)}=0.99$, la covarianza inicial estimada del estado, $P$, se fijó como (39), la covarianza de las perturbaciones asociadas al estado se estableció como $Q=0.01 \cdot P$ y la covarianza de los ruidos asociados a la salida $R$ se determinó como (40).

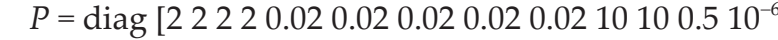
$\left.10^{-6} 0.1\right]$

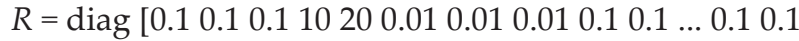
$0.20 .22020200 .20 .2]$

El estimador se programó con el asistente matemático Matlab® (MathWorks 2011) para su simulación, utilizando una PC con 2 GB de memoria RAM, un procesador Intel® Celeron ${ }^{\circledR} 560$ de $2.13 \mathrm{GHz}$ y sistema operativo Windows ${ }^{\circledR}$ 7. Para evaluar el desempeño del estimador se realizó el siguiente experimento. Primero se simuló el funcionamiento de la FCCU según el modelo de referencia durante $600 \mathrm{~s}$, partiendo del estado inicial $x_{0}$ dado por (41) y el vector de entradas constantes $u$ dado por (42), y se registraron los valores del estado y de las salidas del sistema con un período de muestreo de $1 \mathrm{~s}$. Posteriormente se simuló el estimador durante $600 \mathrm{~s}$, partiendo del mismo estado inicial $x_{0}$, con el mismo vector de entradas constantes $u$ y considerando las salidas registradas en la primera parte del experimento, y se registraron los valores del estado estimado con un período de $1 \mathrm{~s}$. Finalmente, se compararon los valores estimados de los estados con los valores de los estados registrados en la primera parte del experimento como referencia.

$x_{0}=[808 \mathrm{~K} 962 \mathrm{~K} 1148 \mathrm{~K} 162 \mathrm{kPa} 204 \mathrm{kPa} 243 \mathrm{kPa} \ldots$

... $279 \mathrm{kPa} 156 \mathrm{kPa}$... $4597 \mathrm{~kg} 124173 \mathrm{~kg} 1618 \mathrm{~kg}$... ... $\left.8.75 \cdot 10^{-4} \mathrm{~kg} / \mathrm{kg} 7.85 \cdot 10^{-3} \mathrm{~kg} / \mathrm{kg} 246 \mathrm{~mol}\right]^{\mathrm{T}}$

$u=\left[\begin{array}{llllllll}1 & 0 & 0 & 0 & 0.95 & 0 & 0 & 0.610 .4213 .8 \mathrm{~kg} / \mathrm{s} 0 \mathrm{~kg} / \mathrm{s} \ldots\end{array}\right.$

... $26 \mathrm{~kg} / \mathrm{s} 5.25 \mathrm{~kg} / \mathrm{s} 34 \mathrm{~kg} / \mathrm{s} 297 \mathrm{~K} 511 \mathrm{~K} 1]^{\mathrm{T}}$

En las gráficas desde la Figura 4 hasta la Figura 12 se muestran los valores estimados de las nueve variables de estado que no son salidas del modelo de la FCCU, así como el error absoluto cometido en estas estimaciones. Los valores de la raíz del error cuadrático medio (RMSE) de las estimaciones de estas nueve variables de estado, calculados según (43), se presentan en la Tabla 6. Puede observarse que la calidad de las estimaciones logradas de estas variables de estado es muy buena, con errores de estimación muy bajos en relación con el rango de variación de las variables de estado respectivas reflejado en las gráficas.
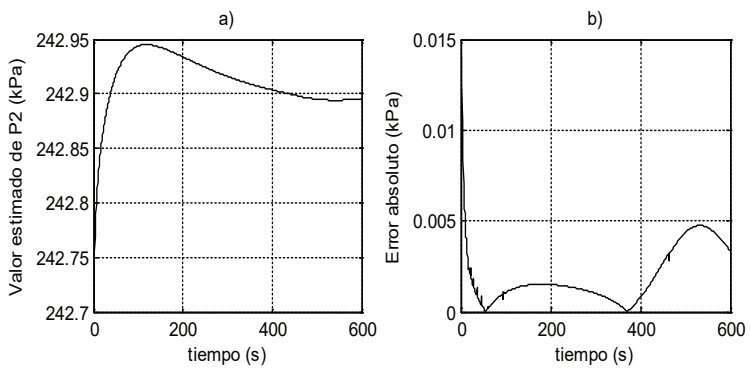

Figura 4. a) Valor estimado de $P 2$, b) error absoluto de estimación

$\mathrm{RMSE}=\sqrt{\frac{\sum_{k=1}^{l}\left(m_{k}-\hat{m}_{k}\right)^{2}}{l}}$ 
donde

$l=600$ es el total de puntos de muestreo registrados en el experimento,

$m_{k}=$ valor de la variable de estado correspondiente al $k$-ésimo punto dado por el modelo de referencia y $\hat{m}_{k}=$ valor de la variable de estado correspondiente al $k$-ésimo punto dado por el estimador.
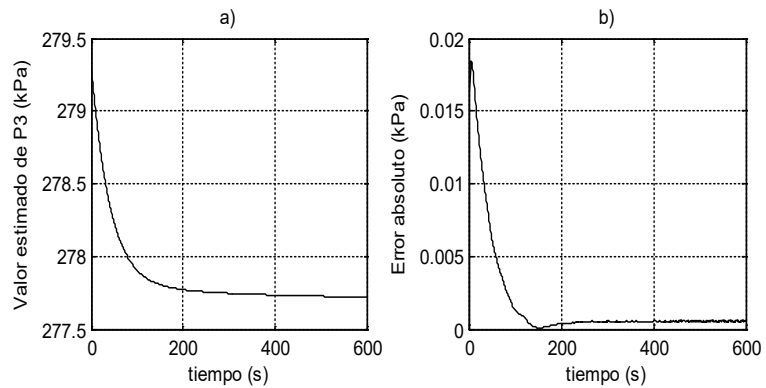

Figura 5. a) Valor estimado de P3, b) error absoluto de estimación
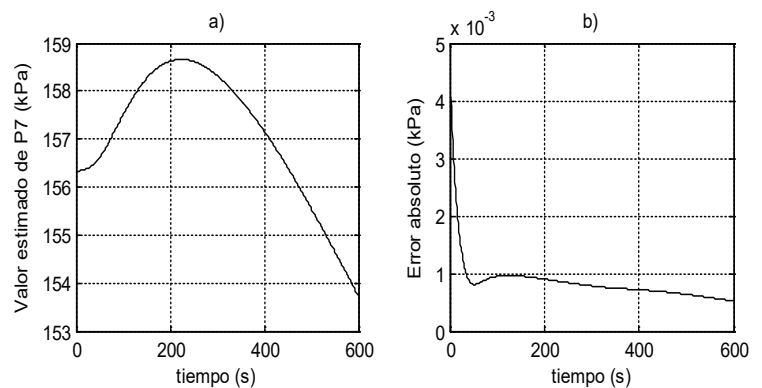

Figura 6. a) Valor estimado de P7, b) error absoluto de estimación
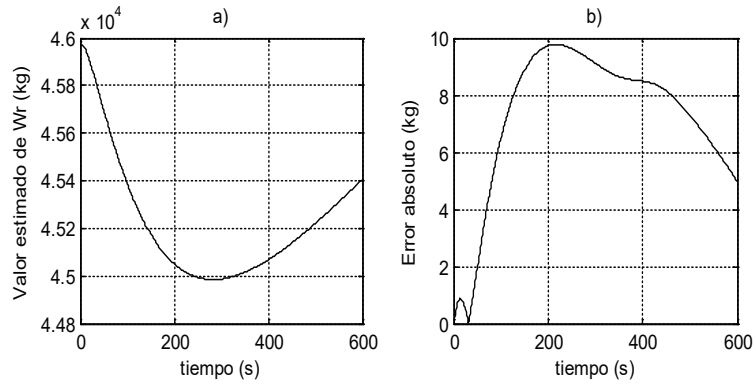

Figura 7. a) Valor estimado de $\mathrm{Wr}$, b) error absoluto de estimación
El tiempo promedio que tarda en la simulación el estimador diseñado para computar una estimación del estado del sistema es de 15.4635 s. Teniendo en cuenta que el experimento se desarrolló con el asistente matemático Matlab® en una PC con sistema operativo Windows ${ }^{\circledR} 7$ y que la dinámica de la FCCU fue relativamente lenta, se puede afirmar que la velocidad de respuesta del estimador es adecuada.
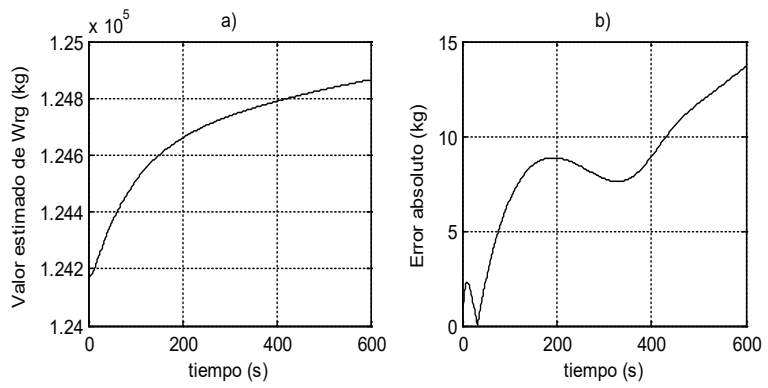

Figura 8. a) Valor estimado de Wrg, b) error absoluto de estimación
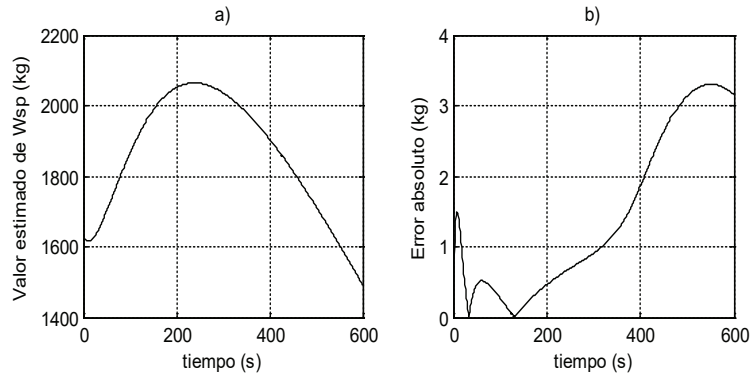

Figura 9. a) Valor estimado de Wsp, b) error absoluto de estimación
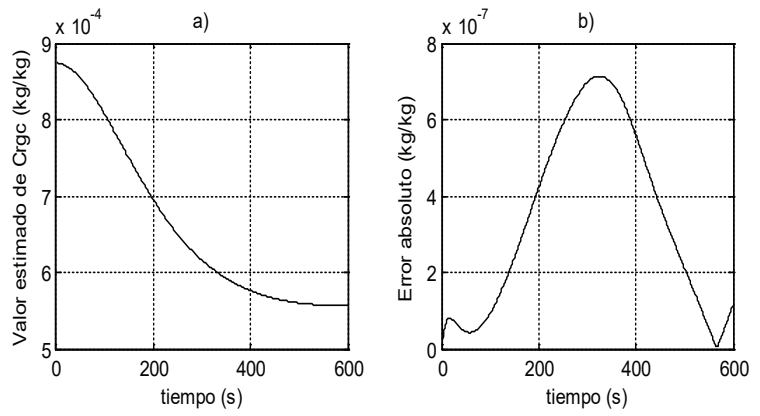

Figura 10. a) Valor estimado de $\mathrm{Crgc}$, b) error absoluto de estimación

Tabla 6. RMSE de las estimaciones de las nueve variables de estado que no son salidas de la FCCU

\begin{tabular}{cccccc}
\hline Variable & RMSE & Variable & RMSE & Variable & RMSE \\
\hline P2 & $2.61 \cdot 10^{-3}(\mathrm{kPa})$ & Wr & $7.7114(\mathrm{~kg})$ & Crgc & $4.15 \cdot 10^{-7}(\mathrm{~kg} / \mathrm{kg})$ \\
P3 & $3.87 \cdot 10^{-3}(\mathrm{kPa})$ & Wrg & $9.0012(\mathrm{~kg})$ & Csc & $1.69 \cdot 10^{-7}(\mathrm{~kg} / \mathrm{kg})$ \\
P7 & $9.41 \cdot 10^{-4}(\mathrm{kPa})$ & Wsp & $1.8308(\mathrm{~kg})$ & $\mathrm{n}$ & $2.6658(\mathrm{~mol})$ \\
\hline
\end{tabular}



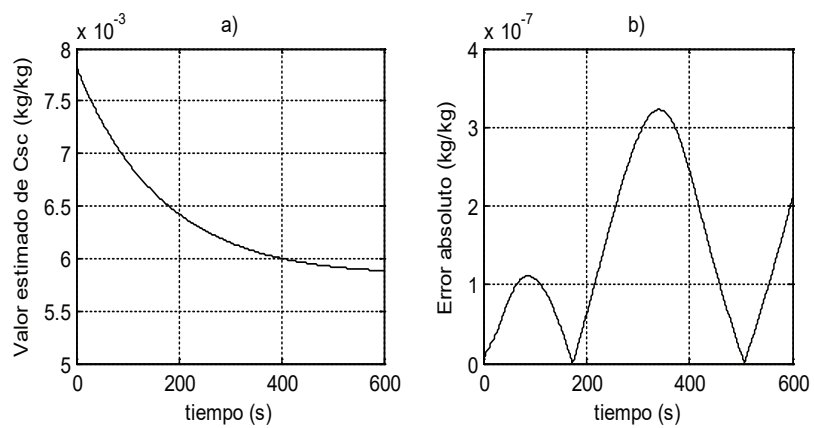

Figura 11. a) Valor estimado de Csc, b) error absoluto de estimación
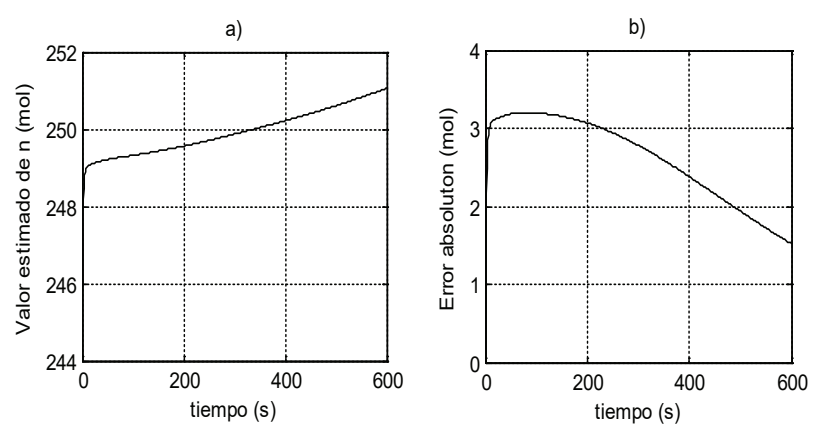

Figura 12. a) Valor estimado de $n$, b) error absoluto de estimación

\section{Conclusiones}

La FCCU es un sistema multivariable de gran complejidad, con fuerte interacción entre sus variables y un comportamiento dinámico acentuadamente no lineal que no está completamente descrito por primeros principios claramente establecidos. De este sistema se han reportado modelos matemáticos de algunos subsistemas y algunos modelos de la FCCU completa, todos de caja blanca o de caja negra, así como estimadores de estados de tipo filtro extendido de Kalman (EKF) y estimador de horizonte deslizante (MHE) basados en modelos de caja blanca. En este trabajo se presentó un modelo gris de una FCCU de tipo IV y se diseñó un estimador de tipo GMUKF empleando dicho modelo gris.

Los resultados de las pruebas realizadas para evaluar el desempeño de este estimador demuestran una satisfactoria estimación de las nueve variables de estado que no son medibles. El tiempo promedio que tarda en la simulación el estimador para computar una estimación del estado denota una adecuada velocidad de respuesta del estimador, considerando que la implementación en hardware de este estimador reduciría mucho el tiempo de obtención de una estimación y que la FCCU se puede controlar en la práctica con períodos de muestreo del orden de varios segundos.

\section{ReferenCias}

Boum A.T., Latifi A., Corriou J.P. Multivariable control and online state estimation of an FCC Unit. Journal of Engineering Science and Technology Review, volumen 8 (número 3), 2015: 158-168.

Dasila P.K., Choudhury I., Saraf D., Chopra S., Dalai A. Parametric sensitivity studies in a commercial FCC Unit. Advances in Chemical Engineering and Science, volumen 2, 2012: 136-149.

Ellis R.C., Li X., Riggs J.B. Modeling and optimization of a model IV fluidized catalytic cracking unit. AIChE Journal, volumen 44 (número 9), 1998: 2068-2079.

Garcia-Dopico M. y Garcia A. Modeling and Simulation of the Fluidized Bed and Freeboard of an FCCU Regenerator. Chemistry and Technology of Fuels and Oils, volumen 52 (número 6), 2017: 716-731.

Han I.S. y Chung C.B. Dynamic modeling and simulation of a fluidized catalytic cracking process. Part I: Process modeling. Chemical Engineering Science, volumen 56 (número 5), 2001: 1951-1971.

Heydari M., AleEbrahim H., Dabir B. Study of seven-lump kinetic model in the fluid catalytic cracking unit. American Journal of Applied Sciences, volumen 7 (número 1), 2010: 71-76.

Huang Y., Dash S., Reklaitis G.V., Venkatasubramanian V. EKF based estimator for FDI in the model IV FCCU, SAFEPROCESS 2000 IFAC Symposium, Budapest, Hungary, 2000.

Julier S.J. y Uhlmann J.K. Unscented filtering and nonlinear estimation. Proceedings of the IEEE, volumen 92 (número 3), 2004: 401-422.

Long J., Mao M.S., Zhao G.Y. Model optimization for an industrial fluid catalytic cracking riser-regenerator unit by differential evolution algorithm. Petroleum Science and Technology, volumen 33 (números 13-14), 2015: 1380-1387.

MathWorks. Matlab. The language of technical computing, The mathworks, Inc., 2011.

McFarlane R.C., Reineman R.C., Bartee J.F., Georgakis C. Dynamic simulator for a model IV fluid catalytic cracking unit. Computers \& Chemical Engineering, volumen 17, 1993: 273-300.

Michalopoulos J., Papadokonstadakis S., Arampatzis G., Lygeros A. Modelling of an industrial fluid catalytic cracking unit using neural networks. Chemical Engineering Research and Design,volumen 79 (número 2), 2001: 137-142.

Ramachandran R., Rangaiah G.P., Lakshminarayanan S. Data analysis, modeling and control performance enhancement of an industrial fluid catalytic cracking unit. Chemical Engineering Science, volumen 62 (número 1), 2007: 958-1973.

Roman R., Nagy Z.K., Cristea M.V., Agachi S.P. Dynamic modelling and nonlinear model predictive control of a fluid catalytic cracking unit. Computers \& Chemical Engineering, volumen 33 (número 3), 2009: 605-617.

Sadeghbeigi R. Fluid Catalytic Cracking Handbook, USA, Butterworth-Heinemann, 2000. 
Souza J.A., Vargas J.V.C., Ordonez J.C., Martignoni W.P., Meien O.F.V. Thermodynamic optimization of fluidized catalytic cracking (FCC) units. International Journal of Heat and Mass Transfer, volumen 54, 2011: 1187-1197.

Tootoonchy H. y Hashemi H.H. Fuzzy logic modeling and controller design for a fluidized catalytic cracking unit, World Congress on Engineering and Computer Science, San Francisco, USA, 2013.

Zhang Y., Lu C., Li T. A practical countercurrent fluid catalytic cracking regenerator model for in situ operation optimization. AIChE Journal, volumen 58 (número 9), 2012: 2770-2784.

\section{Citación sugerida:}

\section{Citación estilo Chicago}

Cepero-Díaz, Ariel Omar, Ana Isabel González-Santos. Estimación de estado de una unidad de craqueo catalítico fluido con filtro desaromatizado de Kalman. Ingeniería Investigación y Tecnología, XIX, 01 (2018): 11-23.

\section{Citación estilo ISO 690}

Cepero-Díaz A.O., González-Santos A.I. Estimación de estado de una unidad de craqueo catalítico fluido con filtro desaromatizado de Kalman. Ingeniería Investigación y Tecnología, volumen XIX (número 1), enero-marzo 2018: 11-23.

\section{SemblanZas de LOS Autores}

Ariel Omar Cepero-Díaz. Obtuvo el título de ingeniero en automática en el Instituto Superior Politécnico José Antonio Echeverría, (CUJAE) - La Habana, Cuba, en 1998 y el de Máster en Automática en el mismo instituto en 2001. Actualmente realiza estudios de doctorado en la temática de estimadores de estados no lineales basados en modelos con aplicación en la industria. Es profesor auxiliar del Departamento de Automática y Computación de la CUJAE, impartiendo asignaturas de la disciplina de sistemas de control, ha sido profesor invitado en universidades de Ecuador y Venezuela.

Ana Isabel González-Santos. Recibió el título de Ingeniería en Máquinas Computadoras por el Instituto Superior Politécnico José Antonio Echeverría, (CUJAE) - La Habana, Cuba, en el año 1994 y el de Máster en Automática en el mismo instituto en 1996. Es doctora por el programa de doctorado tecnologías de la información de la Facultad de Ciencias de la Universidad de Valladolid en España desde el año 2000. Su tesis de doctorado estuvo asociada al control predictivo no lineal de procesos por lotes. Desde el año 2001 sus intereses de investigación han estado relacionados con las aplicaciones de control predictivo no lineal y estimación de estados con significación económica en problemas industriales. Es profesora titular del departamento de Automática y Computación de la CUJAE. Tiene en sus 20 años de vida profesional 19 ingenieros graduados, 3 especialistas, 11 máster en ciencias y 2 tesis. De igual manera, tiene 1 libro publicado, 6 artículos en revistas referenciadas de primer nivel y otro número importante de varias publicaciones en revistas y eventos internacionales en los últimos 5 años. Ha estado como profesora invitada en universidades de Ecuador, Venezuela y México. Es profesora en el Tecnológico de Monterrey, campus Cd Juárez desde el año 2004, donde ha impartido materias relacionadas con los automatismos lógicos, ingeniería de control, informática industrial y comunicaciones industriales. 